\title{
Succession of methanogenic archaea in rice straw incorporated into a Japanese rice field: estimation by PCR-DGGE and sequence analyses
}

\author{
ATSUO SUGANO, ${ }^{1}$ HIDETAKA TSUCHIMOTO, ${ }^{1}$ TUN CHO CHO, ${ }^{1}$ MAKOTO KIMURA ${ }^{1}$ and \\ SUSUMU ASAKAWA ${ }^{1,2}$ \\ ${ }^{1}$ Graduate School of Bioagricultural Sciences, Nagoya University, Nagoya, 464-8601 Japan \\ 2 Corresponding author (asakawa@agr.nagoya-u.ac.jp)
}

Received January 12, 2005; accepted June 20, 2005; published online July 12, 2005

Summary The succession and phylogenetic profiles of methanogenic archaeal communities associated with rice straw decomposition in rice-field soil were studied by polymerase chain reaction-denaturing gradient gel electrophoresis (PCRDGGE) analysis followed by $16 \mathrm{~S}$ rDNA sequencing. Nylon bags containing either leaf sheaths or blades were buried in the plowed layer of a Japanese rice field under drained conditions during the off-crop season and under flooded conditions after transplanting. In addition, rice straw samples that had been buried in the rice field under drained conditions during the offcrop season were temporarily removed during spring plowing and then re-buried in the same rice field under flooded conditions at transplanting. Populations of methanogenic archaea were examined by amplification of the 16S rRNA genes in the DNA extracted from the rice straw samples. No PCR product was produced for samples of leaf sheath or blade prior to burial or after burial under drained conditions, indicating that the methanogen population was very small during decomposition of rice straw under oxic conditions. Many common bands were observed in rice straw samples of leaf sheath and blade during decomposition of rice straw under flooded conditions. Cluster analysis based on DGGE patterns divided methanogenic archaeal communities into two groups before and after the mid-season drainage. Sequence analysis of DGGE bands that were commonly present were closely related to Methanomicrobiales and Rice cluster I. Methanomicrobiales, Rice cluster I and Methanosarcinales were major members before the mid-season drainage, whereas the DGGE bands that characterized methanogenic archaeal communities after the mid-season drainage were closely related to Methanomicrobiales. These results indicate that mid-season drainage affected the methanogenic archaeal communities irrespective of their location on rice straw (sheath and blade) and the previous history of decomposition during the off-crop season.

Keywords: decomposition, methanogen, paddy field soil, phylogenetic profile.

\section{Introduction}

Rice fields are usually flooded during most of the cultivation period. Anaerobic decomposition of organic matter in flooded rice fields produces methane, making the fields a major source of atmospheric methane. Rice straw is commonly incorporated into rice fields to maintain soil fertility, especially following the use of combine harvesters. Previous investigations reported that application of rice straw to rice fields increased methane emissions (Schütz et al. 1989, Yagi and Minami 1990, Sass et al. 1991, Chidthaisong et al. 1996, Watanabe et al. 1998), and the emissions increased in proportion to the amount of rice straw applied (Watanabe et al. 1995). Acetate and syntrophically degraded propionate are the main precursors of methane during decomposition of rice straw in anoxic rice-field soils (Glissmann and Conrad 2000).

The dynamics of methanogenic populations and their activities in rice fields amended with rice straw have been investigated (Adachi et al. 1996, Joulian et al. 1996, Asakawa et al. 1998, Kaku et al. 2000). In recent years, molecular biological methods have been applied to elucidate microbial community structures. Methanogenic archaeal communities involved in the decomposition of rice straw in anoxic rice soil microcosms have been determined by sequencing rRNA gene libraries, terminal restriction fragment length polymorphisms (T-RFLP) and slot blot hybridizations to include Methanobacterium, Methanosarcina and Rice cluster I (Weber et al. 2001). However, the methanogenic archaeal communities responsible for decomposition of rice straw in fields have not yet been investigated using molecular biological methods.

In a previous study, the bacterial communities responsible for decomposition of rice straw incorporated into a rice field were examined by denaturing gradient gel electrophoresis (DGGE) analysis (Sugano et al. 2005). The bacterial communities were mainly affected by the part of straw (sheath or blade) that was buried. Major bacterial groups found in the rice field under both flooded and drained conditions were Proteobacteria, the CFB group and Spirochaetes. Microscopic observation of the decomposition process revealed that the leaf blade decomposed faster than the leaf sheath, and that the de- 
composition of the leaf blade proceeded mainly from inside the tissue. In contrast, the leaf sheath underwent decomposition from both the inside of the tissue and from the surface (Kimura and Tun 1999, Tun and Kimura 2000).

The present study sought to elucidate, by DGGE, the succession and phylogenetic profile of methanogenic archaeal communities during the decomposition of rice straw buried in a rice field under flooded and drained conditions. The same rice straw samples as those studied by Tun and Kimura (2000), Tun et al. (2002), Nakamura et al. (2003) and Sugano et al. (2005) were used. Thus, it was possible to compare the characteristics of methanogenic archaeal and bacterial communities during the decomposition process.

\section{Materials and methods}

\section{Experimental field}

The field incubations were performed in a paddy field at Aichi-ken Anjo Research and Extension Center, central Japan $\left(34^{\circ} 8^{\prime} \mathrm{N}, 137^{\circ} 5^{\prime} \mathrm{E}\right)$. The soil had a total $\mathrm{C}$ content $=12.8 \mathrm{~g}$ $\mathrm{kg}^{-1}$; total $\mathrm{N}$ content $=1.1 \mathrm{~g} \mathrm{~kg}^{-1} ; \mathrm{pH}\left(\mathrm{H}_{2} \mathrm{O}\right)$ value $=6.3$; amorphous Fe content $=3.76 \mathrm{~g} \mathrm{~kg}^{-1}$; and clay content $=230 \mathrm{~g}$ $\mathrm{kg}^{-1}$. The soil type is Oxyaquic Dystrudept.

\section{Rice straw samples}

Rice straw (Oryza sativa L. cv. 'Koshihikari') was collected from the Aichi-ken Anjo Research and Extension Center, at harvest time. Five-cm segments of leaf sheath and leaf blade were buried separately in nylon mesh bags (1-mm mesh size). Eighteen and 24 bags of sheaths ( 5 pieces per bag) and leaf blades (20 pieces per bag), respectively, were buried horizontally in the rice field at 5- to 10-cm depths: on June 19, 1998, 2 days after transplanting under flooded conditions (Experiment 1), and on November 12, 1998, after the harvest of rice plants under drained conditions (Experiment 2). To allow for plowing, the unsampled bags in Experiment 2 were temporarily removed on April 14, 1999 and reburied on May 5, 1999 after transplanting rice seedlings under flooded conditions. The field management procedure during the experimental period has been described previously (Tun et al. 2002). The field was flooded in early June 1998 and in late April 1999, drained in early October 1998 and late August 1999, and left fallow during the off-crop season in winter (from November 1998 to April 1999). Mid-season drainage was performed from July 22 to August 9, 1998 and from June 14 to July 2, 1999. Mean temperature ranges were $19-30{ }^{\circ} \mathrm{C}$ (June to August 1998), $8-26{ }^{\circ} \mathrm{C}$ (September to November 1998), $2-10{ }^{\circ} \mathrm{C}$ (December 1998 to February 1999), 7-20 ${ }^{\circ} \mathrm{C}$ (March to May 1999) and 21-29 ${ }^{\circ} \mathrm{C}$ (June to August 1999). The rainfall in winter from November to April was $337 \mathrm{~mm}$, and the annual rainfall was 1500-1900 mm.

\section{Date of sampling}

Three nylon mesh bags each of leaf sheaths and leaf blades were removed at each sampling time. In Experiment 1, sam- ples were collected during the period of rice cultivation on July 8 and 22, August 4, September 2 and 18, and October 16, 1998. In Experiment 2, samples were collected under drained conditions on December 9, 1998, February 25, 1999 and April 14 and during the period of rice cultivation on June 15 and 29, July 13 and 28, and August 9, 1999. The nylon mesh bags were gently washed with distilled water to remove soil particles and plant roots and were then frozen at $-20{ }^{\circ} \mathrm{C}$ until analysis.

\section{DNA extraction}

Samples of leaf sheaths and blades ( $0.5 \mathrm{~g}$ fresh mass) for DNA analysis were obtained from the middle section of several rice straw segments. The DNA extraction was performed by the freeze- thaw method (Zhou et al. 1996, Tun et al. 2002). Some of the extracted DNA samples were further purified on a Sephadex G-200 gel filtration column (Jackson et al. 1997, Cahyani et al. 2003).

\section{PCR amplification and DGGE analysis}

Methanogenic archaeal 16S rDNA was amplified with the primer set consisting of a 0357F-GC clamp (Eschericha coli position: 340-357, 5' -CGC CCG CCG CGC GCG GCG GGC GGG GCG GGG GCA CGG GGGG CCC TAC GGG GCG CAG CAG-3', where the underlined sequence corresponds to the GC clamp) and 0691R (E. coli position: 707-691, 5'-GGA TTA CAR GAT TTC AC-3'; Watanabe et al. 2004). The PCR was performed in a total volume of $50 \mu \mathrm{l}$, containing $75 \mathrm{pmol}$ of each primer, $0.25 \mathrm{mM}$ dNTP mixture, $1 \times E x$ Taq buffer (with $20 \mathrm{mM} \mathrm{Mg}^{2+}$; TaKaRa, Tokyo, Japan), $2.5 \mathrm{U}$ Ex Taq DNA polymerase (TaKaRa), and about $20 \mathrm{ng}$ of DNA template. The amplification condition was $94{ }^{\circ} \mathrm{C}$ for $3 \mathrm{~min}$ (initial denaturation), followed by 35 cycles of denaturation at $94{ }^{\circ} \mathrm{C}$ for $1 \mathrm{~min}$, annealing at $53^{\circ} \mathrm{C}$ for $1 \mathrm{~min}$, extension at $72{ }^{\circ} \mathrm{C}$ for $2 \mathrm{~min}$, and a final extension at $72{ }^{\circ} \mathrm{C}$ for $8 \mathrm{~min}$ in a TaKaRa PCR Thermal Cycler (TaKaRa). The PCR products were separated on an $8 \%(\mathrm{w} / \mathrm{v})$ polyacrylamide gel with a denaturing gradient from 25 to $65 \%$ (100\% denaturant, $7 \mathrm{M}$ urea and $40 \%$ (v/v) formamide) as described by Cahyani et al. (2004).

\section{Statistical analysis}

Cluster and principal component analyses were performed for the DGGE data based on the position and the intensity of bands observed $(0=$ no band; $1=$ weak; $2=$ moderate; $3=$ strong). The values were normalized for both analyses. Principal component analysis was performed with Excel statistics 97 for Windows (SRI, Tokyo, Japan) and a correlation matrix. Cluster analysis was performed with a Black box program (Aoki 1996) by the Ward method, which is one of the hierarchical techniques (agglomerative type) that can usually form obvious clusters. Analysis of Molecular Variance (AMOVA) was conducted to compare the DGGE patterns of the communities. The calculation of $\Phi_{\mathrm{ST}}$ (the analogue of $F_{\mathrm{ST}}$ in the analysis of variance (ANOVA)) and a significance test for the $\Phi_{\mathrm{ST}}$ value based on the permutation procedure were performed according to Excoffier et al. (1992). 


\section{DNA sequencing of DGGE bands}

Characteristic DGGE bands were excised from at least two lanes of the gel and their electrophoretic mobility was confirmed to be the same as the original one by DGGE, as described above. The DNA was sequenced directly. The few bands that could not be sequenced directly were cloned and then sequenced (Cahyani et al. 2003). Sequencing was conducted with the DYEnamic ET Terminator Cycle Sequencing Kit according to the manufacturer's instructions (Amersham, Tokyo, Japan) with a 373S DNA Sequencer (ABI, Chiba, Japan).

\section{Phylogenetic analysis}

The close relatives and phylogenetic affiliations of the sequences were determined with the BLAST search program at the DNA Data Bank of Japan (http//:www.ddbj.nig.ac.jp/ E-mail/homology.html). Selected sequences were aligned with Clustal X software, and phylogenetic trees were constructed using the neighbor joining method with the nj plot and TreeView software. Bootstrap analyses were performed with 1000 replications.

\section{Nucleotide sequence accession numbers}

The sequences of the 16S rRNA genes of the methanogenic archaea in this study are available from the DDBJ database. The accession numbers are AB200334 to AB200351.

\section{Results and discussion}

Methanogenic archaeal 16S rRNA genes were successfully amplified by PCR from rice straw samples under flooded con- ditions in both Experiments 1 and 2. However, no PCR poduct was produced by sheath and blade samples before burial or after burial under drained conditions in Experiment 2, indicating that these samples contained little methanogenic archaeal DNA. As bacterial 16S rDNA was successfully amplified from all of the rice straw samples in our previous study (Sugano et al. 2005), we conclude that the amount and purity of the extracted DNAs was sufficient for PCR amplification. Cahyani et al. (2004) detected methanogenic archaea in air-dried rice straw materials and attributed their presence to soil contamination. Using the most probable number (MPN) method, Asakawa et al. (1998) consistently detected methanogens in the drained rice-field soil containing rice straw during the wheat-cropping period. However, our results indicate that methanogens did not grow in rice straw newly buried in drained oxic soil. This was expected because methanogenic archaea require strictly anaerobic conditions for growth.

\section{DGGE patterns of methanogenic archaeal communities in rice straw during decomposition in flooded rice-field soil}

The PCR products of methanogenic archaeal genes were obtained only from samples incubated under flooded conditions. The DGGE patterns of methanogenic archaeal communities in decomposing rice straw under flooded conditions are shown in Figure 1. The DGGE patterns of both leaf sheath and blade samples in Experiments 1 and 2 changed after the mid-season drainage (after August 4 in Experiment 1 and after June 29 in Experiment 2). In total, bands with 24 different mobilities were detected. There was no difference in the total number of DGGE bands between the leaf sheath (17 on average) and the leaf blade (16 on average), or between Experiments 1 (17 on average) and 2 (17 on average). The number of bands repre-

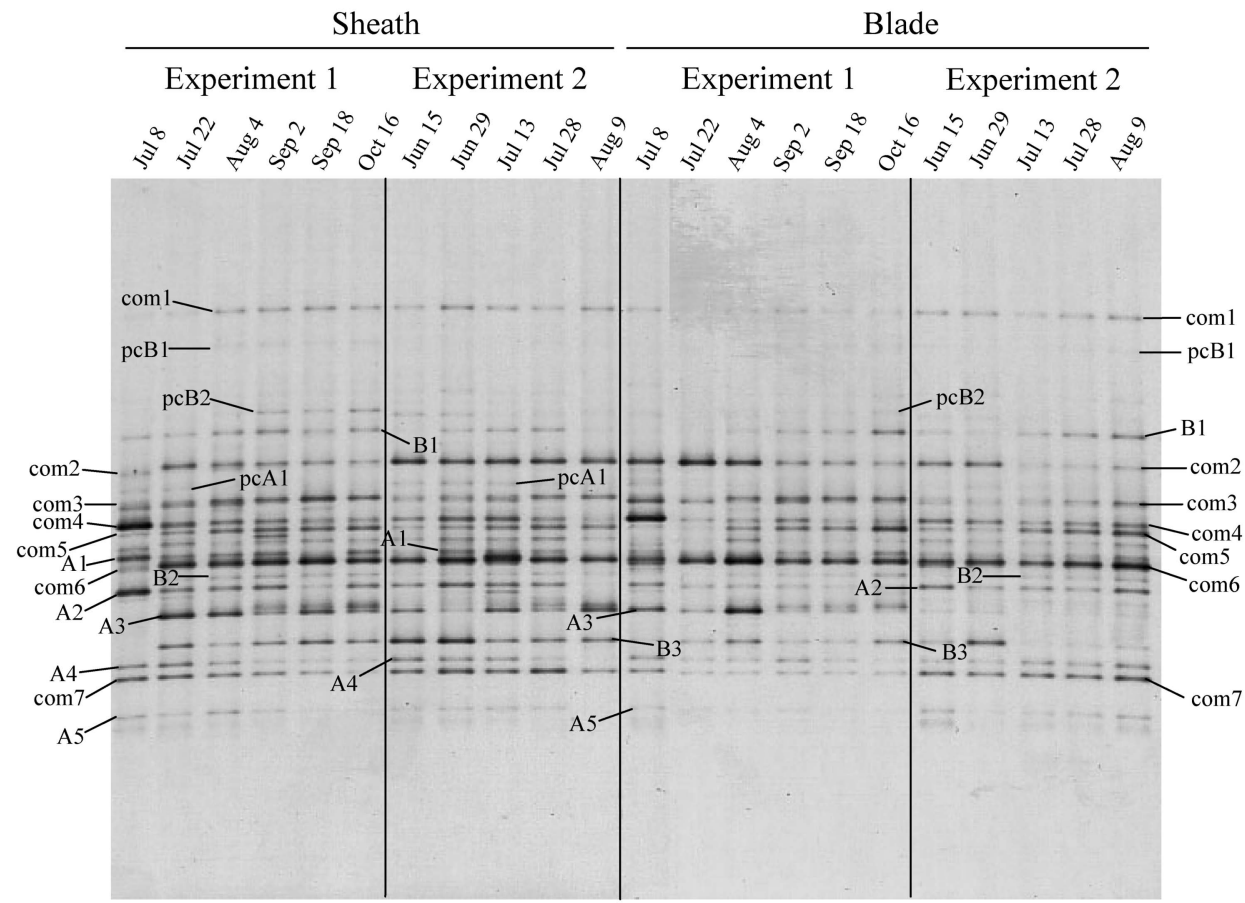

Figure 1. Denaturing gradient gel electrophoresis (DGGE) patterns of methanogenic archaeal communities associated with rice straw decomposition. Bands labeled "com" were present in all samples. Bands $\mathrm{A}$ and $\mathrm{B}$ existed in all samples in Clusters A and B, respectively (see Figure 2). Bands pcA and $\mathrm{pcB}$ denote the bands that characterized Clusters A and B, respectively, in the principal component analysis (see Figure 3 ). The periods of mid-season drainage were from July 22 to August 9, 1998 and from June 14 to July 2, 1999 in Experiments 1 and 2 , respectively. 


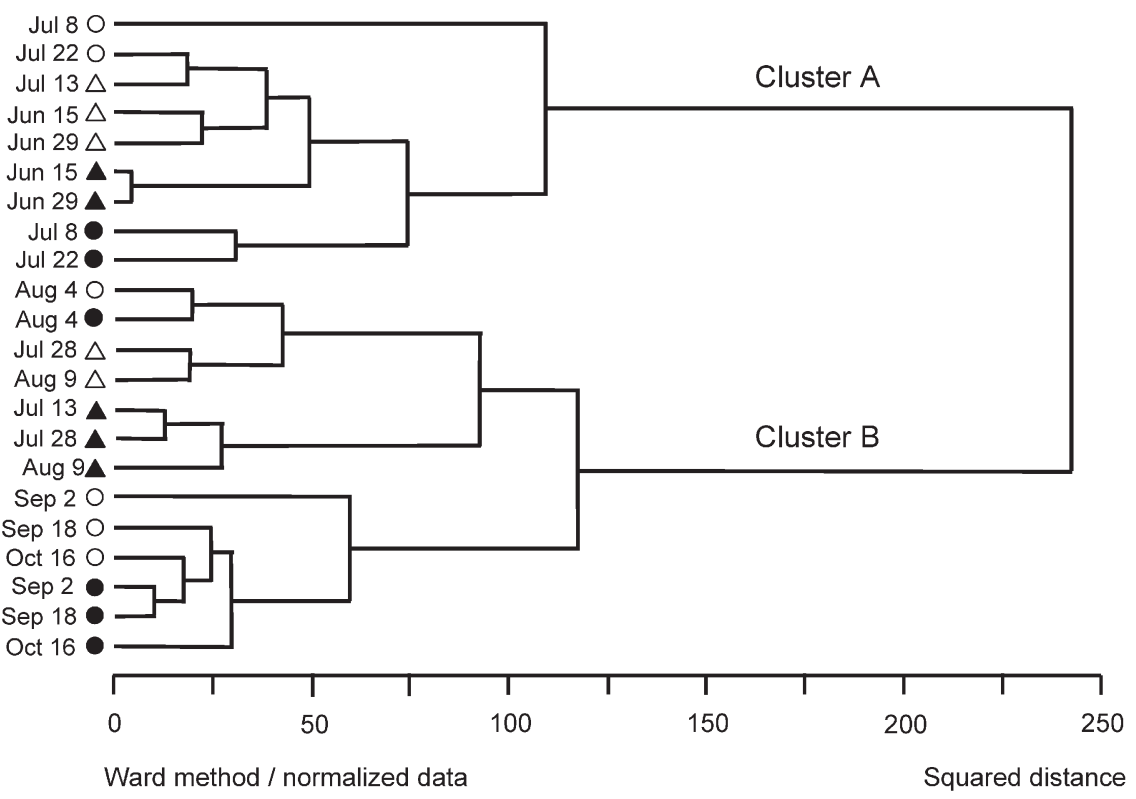

Figure 2. Cluster analysis of DGGE patterns of methanogenic archaeal communities associated with rice straw decomposition. Symbols: $\bigcirc=$ sheath in Experiment 1; $\triangle=$ sheath in Experiment 2; $=$ blade in Experiment 1; and $\boldsymbol{\Lambda}$ = blade in Experiment 2. senting methanogenic archaea during the decomposition of rice straw was slightly higher in our study than in the study of Cahyani et al. (2004) (12 DGGE bands), but similar to the number found in rice roots grown in flooded soil (about 15 bands; Ikenaga et al. 2004). The similar number of DGGE bands in the leaf sheath and leaf blade indicates that methanogenic archaeal communities of similar diversity were located on all parts of the rice straw. In contrast, the diversity of bacterial communities varies between the leaf sheath and blade (Sugano et al. 2005). Seven bands were found in all incubations of the sheath and blade samples in Experiments 1 and 2 (Figure 1). No bands were specific to only the leaf sheath or leaf blade. These findings indicate that the part of rice straw buried had little effect on the methanogenic archaeal communities under flooded conditions.

\section{Statistical analysis of DGGE patterns of methanogenic archaeal communities}

Cluster analysis separated the methanogenic archaeal communities into two groups, Clusters A and B. These clusters corresponded to samples taken before and after mid-season drainage (Figure 2). The methanogenic archaeal communities belonging to Clusters A and B were significantly different $(P<0.001)$ according to the AMOVA of the DGGE banding patterns. Cluster A included sheath and blade samples from before mid-season drainage on July 22 in Experiment 1 and before the end of mid-season drainage on July 2 in Experiment 2. Cluster B included the remaining samples. These differences were significant $(P<0.001)$ when samples obtained during mid-season drainage were excluded. Moreover, bands A1, A2, A3, A4 and A5 and bands B1, B2 and B3 were common in

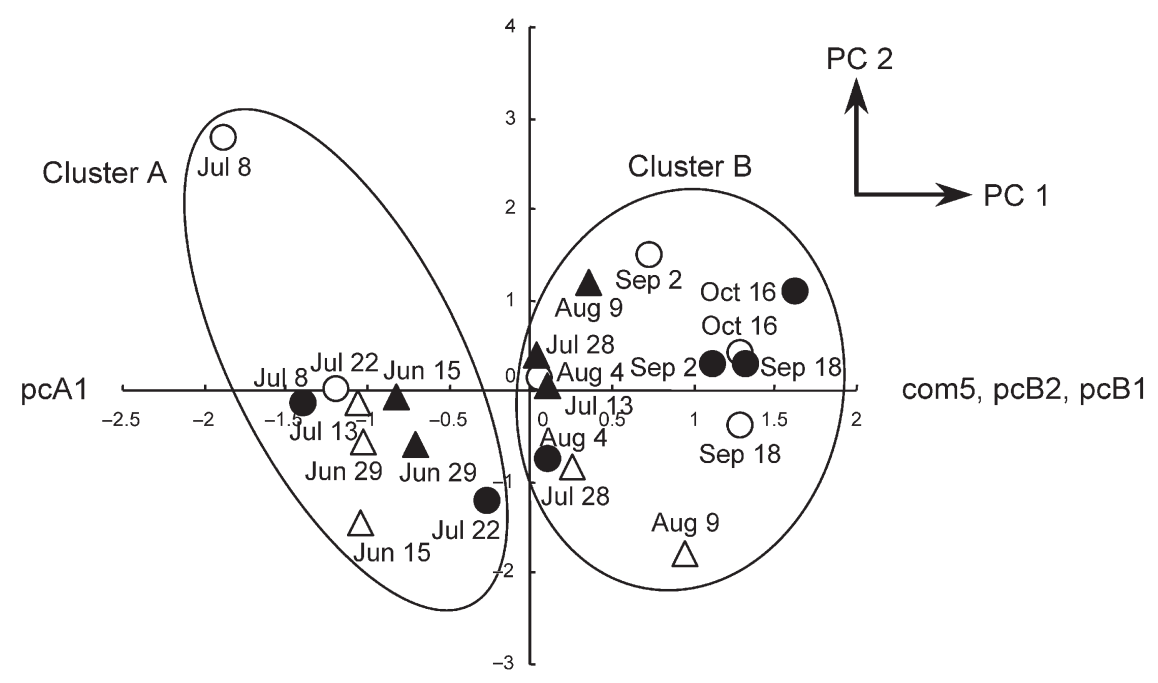

Figure 3. Principal component analysis of DGGE patterns of methanogenic archaeal communities associated with rice straw decomposition. Abbreviations: PC $1=$ first principal component; $\mathrm{PC} 2$ = second principal component. Symbols: $\bigcirc=$ sheath in Experiment 1; $\triangle$ = sheath in Experiment 2; = blade in Experiment 1; and $\boldsymbol{\Lambda}$ = blade in Experiment 2 . 


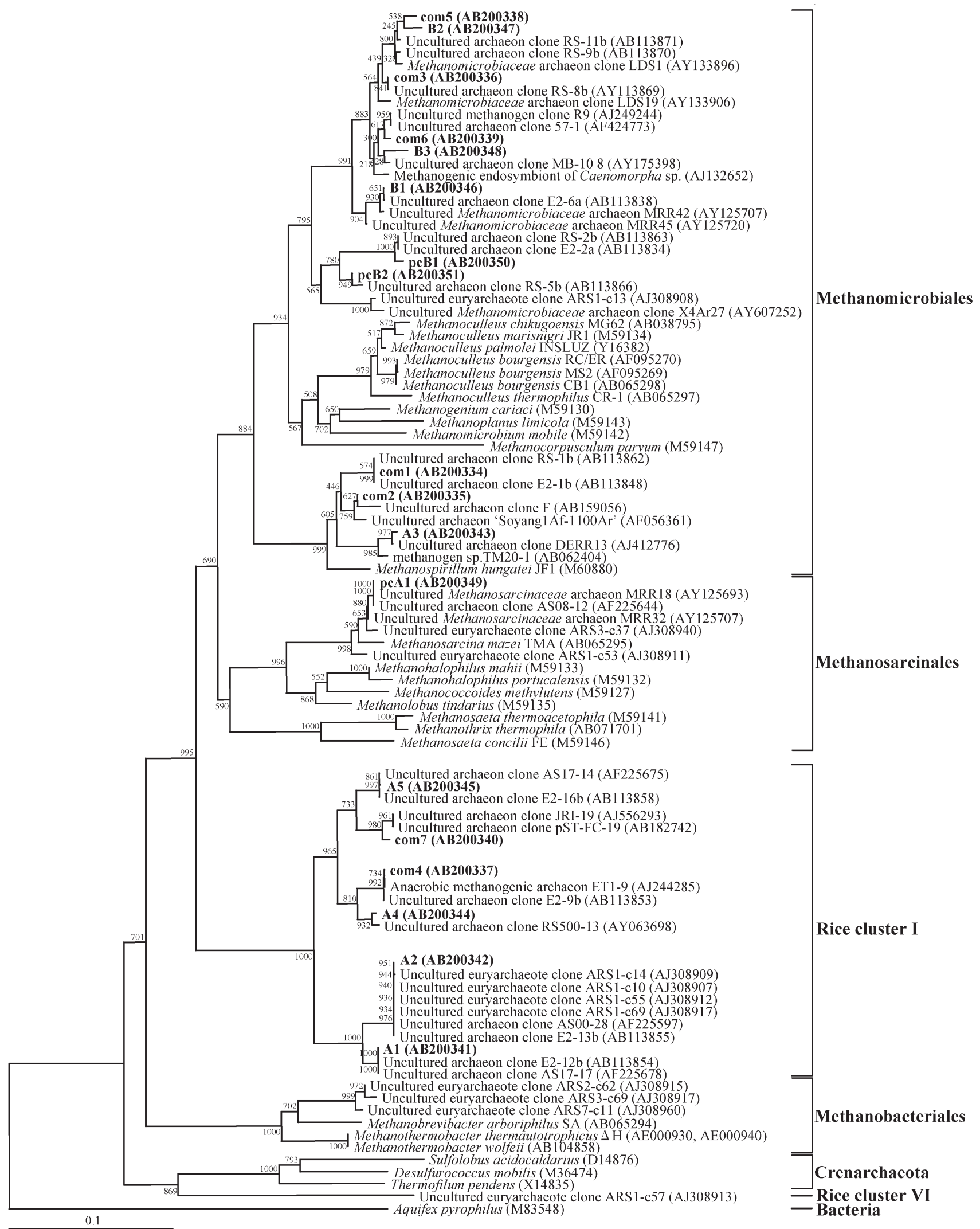

Figure 4. Phylogenetic relationships of methanogenic archaeal 16S rDNA sequences of characterized DGGE bands. The sequences obtained in the present study are shown in bold. Bootstrap values greater than 500 (out of 1000 replicates) are shown at branch points. Aquifex pyrophilus was used as an outgroup. The scale bar represents 0.1 substitutions per nucleotide. Accession numbers of sequences are indicated in parentheses. 
all samples in Clusters A and B, respectively (Figure 1). These results indicate that mid-season drainage has a significant effect on methanogenic archaeal communities associated with rice straw decomposition in rice-field soil.

In contrast, bacterial communities associated with decomposition of rice straw were mainly affected by the part of the straw (leaf sheath or blade) buried (Sugano et al. 2005). The difference in the factors affecting bacterial and methanogenic archaeal communities in decomposition of rice straw may be explained as follows: bacterial communities were directly responsible for decomposition of rice straw, whereas methanogenic archaeal communities did not use biopolymers of rice straw directly, but used only the fermentation end products of decomposition. Glissmann and Conrad (2000) investigated the fermentation pattern during methanogenic decomposition of stem, sheath and blade in an anoxic rice-field soil microcosm and found different fermentation patterns in the early decomposition stages, but no differences in the later methanogenic decomposition stage. Because the methanogenic communities used the same fermentation end products, it may be expected that their communities are unaffected by the differences in bacterial decomposition. Bacterial communities comprising members with diversified substrate requirements and with various respiratory strategies (strictly aerobic, facultatively anaerobic and strictly anaerobic) may be mainly controlled by the composition of rice straw. In contrast, methanogenic archaeal communities can metabolize only a few substrates and may be controlled simply by the oxidation-reduction status.

The principal component analysis is shown in Figure 3. The first and second principal components (PC) contributed 30 and $17 \%$, respectively. The first PC divided the methanogenic archaeal community into two groups, which corresponded to Clusters A or B. Score plots of the samples shifted from the negative load to the positive load in the first PC. The distance of shift during rice cultivation was larger in Experiment 1 than in Experiment 2. Band pcA1 and bands com5, pcB2 and pcB1 characterized Clusters A and B, respectively.

\section{Identification of characteristic DGGE bands}

The DGGE bands were closely related to previously cultured and uncultured methanogens (Figure 4). All sequenced bands that were excised from two lanes and possessed the same mobilities had identical sequences. Bands com1, com2, com3, com5 and com6 were related to the Methanomicrobiales. Bands com 4 and com7 belonged to Rice cluster I. Bands A1, A2, A4 and A5 also belonged to Rice cluster I, and only band A3 belonged to Methanomicrobiales. Bands B1, B2 and B3 also belonged to Methanomicrobiales. Band pcA1, which was specific to Cluster A, belonged to Methanosarcinales, and the closest relatives of Cluster B-specific bands, pcB1, pcB2 and com5, were members of the Methanomicrobiales. Many bands were closely related to uncultured methanogens obtained from rice-field soils, and about a half of the sequenced bands (eight bands) were closely related to those obtained from the same rice-field soil (Watanabe et al. 2004) or rice straw material (Cahyani et al. 2004). Band A2 was closely related to a
Rice cluster I clone obtained from rice straw decomposed in an anoxic rice soil microcosm (Weber et al. 2001). Based on analyses of slot blot hybridizations, Weber et al. (2001) estimated that Rice cluster I was both the most abundant and most active group, although it was a minor group in the cloning and sequencing analyses. On the other hand, members of Methanomicrobiales were a minor group during decomposition of rice straw in their study (Weber et al. 2001). Differences between the results of the present study and those of Weber et al. (2001) may be associated with differences in soil type (sampling region) or experimental conditions.

Our results indicate that the Methanomicrobiales and Rice cluster I are major members in methanogenic archaeal communities associated with decomposition of rice straw. Members of the Methanomicrobiales were more abundant than those of Rice cluster I. Most bands belonging to Rice cluster I were found in Cluster A. Thus, members of Methanomicrobiales, Rice cluster I and Methanosarcinales must have colonized rice straw mainly from the early flooded period to the mid-season drainage, and then been replaced by members of Methanomicrobiales after the mid-season drainage. Ueki et al. (1995) and Kaku et al. (2000) found that the methanogenic activities of rice straw were lower after mid-season drainage than before it. Mid-season drainage may have affected the production rate and the concentration of methanogenic substrates, which resulted in changes in methanogenic activities as well as in structural changes in methanogenic archaeal communities. Band pcA1 (Methanosarcinales), which characterized the methanogenic communities before mid-season drainage, disappeared after mid-season drainage. Wu and Conrad (2001) reported that populations of Methanosarcina in cellulose-degrading methanogenic microbial communities decreased following aeration of anoxic rice field soil, indicating that members of Methanosarcinales in methanogenic archaeal communities were affected by the oxic conditions associated with mid-season drainage.

In conclusion, methanogenic archaeal communities involved in the decomposition of rice straw under flooded conditions were affected by mid-season drainage irrespective of their location on the rice straw (leaf sheath or leaf blade). Methanogenic archaeal populations in rice straw under oxic conditions were either absent or very small. The major methanogenic archaeal groups responsible for decomposition of rice straw under flooded conditions were Methanomicrobiales and Rice cluster I. Methanomicrobiales, Rice cluster I and Methanosarcinales comprised the methanogenic archaeal communities on rice straw before the mid-season drainage, whereas the methanogenic communities on rice staw after the mid-season drainage comprised only the Methanomicrobiales.

\section{Acknowledgments}

We thank T. Watanabe of the Graduate School of Bioagricultural Sciences, Nagoya University for his technical assistance and valuable comments and the staff members of Aichi-ken Anjo Research and Extension Center for allowing us to use the experimental field for rice straw sampling. We thank A. Nakanishi, T. Torimaru and N. Tomaru 
of the Graduate School of Bioagricultural Sciences, Nagoya University for their help in the AMOVA analysis.

\section{References}

Adachi, K., G. Oyediran and T. Senboku. 1996. Effect of application of rice straw and cellulose on methane emission and biological nitrogen fixation in a subtropical paddy field. II. Enumeration of populations of methanogenic bacteria by most probable number method and roll tube method. Soil Sci. Plant Nutr. 42:713-723.

Aoki, S. 1996. http://aoki2.si.gunma-u.ac.jp/bb0/BlackBox0.html.

Asakawa, S., M. Akagawa-Matsushita, Y. Koga and K. Hayano. 1998. Communities of methanogenic bacteria in paddy field soils with long-term application of organic matter. Soil Biol. Biochem. 30:299-303.

Cahyani, V.R., K. Matsuya, S. Asakawa and M. Kimura. 2003. Succession and phylogenetic composition of bacterial communities responsible for the composting process of rice straw estimated by PCR-DGGE analysis. Soil Sci. Plant Nutr. 49:619-630.

Cahyani, V.R., K. Matsuya, S. Asakawa and M. Kimura. 2004. Succession and phylogenetic profile of methanogenic archaeal communities during the composting process of rice straw estimated by PCR-DGGE analysis. Soil Sci. Plant Nutr. 50:555-563.

Chidthaisong, A., K. Inubushi, Y. Muramatsu and I. Watanabe. 1996. Production potential and emission of methane in flooded rice soil microcosms after continuous application of straw. Microb. Environ. 11:73-78.

Excoffier, L., P.E. Smouse and J.M. Quattro. 1992. Analysis of molecular variance inferred from metric distances among DNA haplotypes: application to human mitochondrial DNA restriction data. Genetics 131:479-491.

Glissmann, K. and R. Conrad. 2000. Fermentation pattern of methanogenic degradation of rice straw in anoxic paddy soil. FEMS Microbiol. Ecol. 31:117-126.

Ikenaga, M., S. Asakawa, Y. Muraoka and M. Kimura. 2004. Methanogenic archaeal communities in rice roots grown in flooded soil pots: estimation by PCR-DGGE and sequence analyses. Soil Sci. Plant Nutr. 50:701-711.

Jackson, C.R., J.P. Harper, D. Willoughby, E.E. Roden and P.F. Churchill. 1997. A simple, efficient method for the separation of humic substances and DNA from environmental samples. Appl. Environ. Microbiol. 63:4993-4995.

Joulian, C., B. Ollivier, H.-U. Neue and P.A. Roger. 1996. Microbiological aspects of methane emission by a rice field soil from the Camargue (France): 1. Methanogenesis and related microflora. Eur. J. Soil Biol. 32:61-70.

Kaku, N., A. Ueki, H. Fujii and K. Ueki. 2000. Methanogenic activities on rice roots and plant residue and their contributions to methanogenesis in wetland rice field soil. Soil Biol. Biochem. 32: 2001-2010.

Kimura, M. and C.C. Tun. 1999. Microscopic observation of the decomposition process of leaf sheath of rice straw and colonizing microorganisms during the cultivation period of paddy rice. Soil Sci. Plant Nutr. 45:427-437.
Nakamura, A., C.C. Tun, S. Asakawa and M. Kimura. 2003. Microbial community responsible for the decomposition of rice straw in a paddy field: estimation by phospholipids fatty acid analysis. Biol. Fertil. Soils 38:288-295.

Sass, R.L., F.M. Fisher and P.A. Harcombe. 1991. Mitigation of methane emissions from rice fields: possible adverse effects of incorporated rice straw. Global Biogeochem. Cycles 5:275-287.

Schütz, H., A. Holzapfel-Pschorn, R. Conrad, H. Rennenberg and W. Seiler. 1989. A 3-year continuous record on the influence of daytime, season, and fertilizer treatment on methane emission rates from an Italian rice paddy. J. Geophys. Res. 94:16,405-16,416.

Sugano, A., H. Tsuchimoto, C.C. Tun, S. Asakawa and M. Kimura. 2005. Succession and phylogenetic profile of eubacterial communities in rice straw incorporated into a rice field: estimation by PCR-DGGE analysis. Soil Sci. Plant Nutr. 51:51-60.

Tun, C.C. and M. Kimura. 2000. Microscopic observation of the decomposition process of leaf blade of rice straw and colonizing microorganisms in a Japanese paddy field soil during the cultivation period of paddy rice. Soil Sci. Plant Nutr. 46:127-137.

Tun, C.C., M. Ikenaga, S. Asakawa and M. Kimura. 2002. Community structure of bacteria and fungi responsible for rice straw decomposition in a paddy field estimated by PCR-RFLP analysis. Soil Sci. Plant Nutr. 48:805-813.

Ueki, A., T. Ishida, K. Ohbuchi, N. Kaku, T. Nagano, K. Ueki and T. Wagatsuma. 1995. Effect of organic matter application on growth of rice plants, soil environments and methane emission from paddy fields. Environ. Sci. 8:293-303. In Japanese with English summary.

Watanabe, A., Y. Satoh and M. Kimura. 1995. Estimation of the increase in methane emission from paddy soils by rice straw application. Plant Soil 173:225-231.

Watanabe, A., M. Yoshida and M. Kimura. 1998. Contribution of rice straw carbon to $\mathrm{CH}_{4}$ emission from rice paddies using ${ }^{13} \mathrm{C}$-enriched rice straw. J. Geophys. Res. 103D:8237-8242.

Watanabe, T., S. Asakawa, A. Nakamura, K. Nagaoka and M. Kimura. 2004. DGGE method for analyzing 16 S rDNA of methanogenic archaeal community in paddy field soil. FEMS Microbiol. Lett. 232:153-163.

Weber, S., T. Lueders, M.W. Friedrich and R. Conrad. 2001. Methanogenic populations involved in the degradation of rice straw in anoxic paddy soil. FEMS Microbiol. Ecol. 38:11-20.

Wu, X.L. and R. Conrad. 2001. Functional and structural response of a cellulose-degrading methanogenic microbial community to multiple aeration stress at two different temperatures. Environ. Microbiol. 3:355-362.

Yagi, K. and K. Minami. 1990. Effect of organic matter application on methane emission from some Japanese paddy fields. Soil Sci. Plant Nutr. 36:599-610.

Zhou, J., M.A. Bruns and J.M. Tiedje. 1996. DNA recovery from soils of diverse composition. Appl. Environ. Microbiol. 62:316-322. 

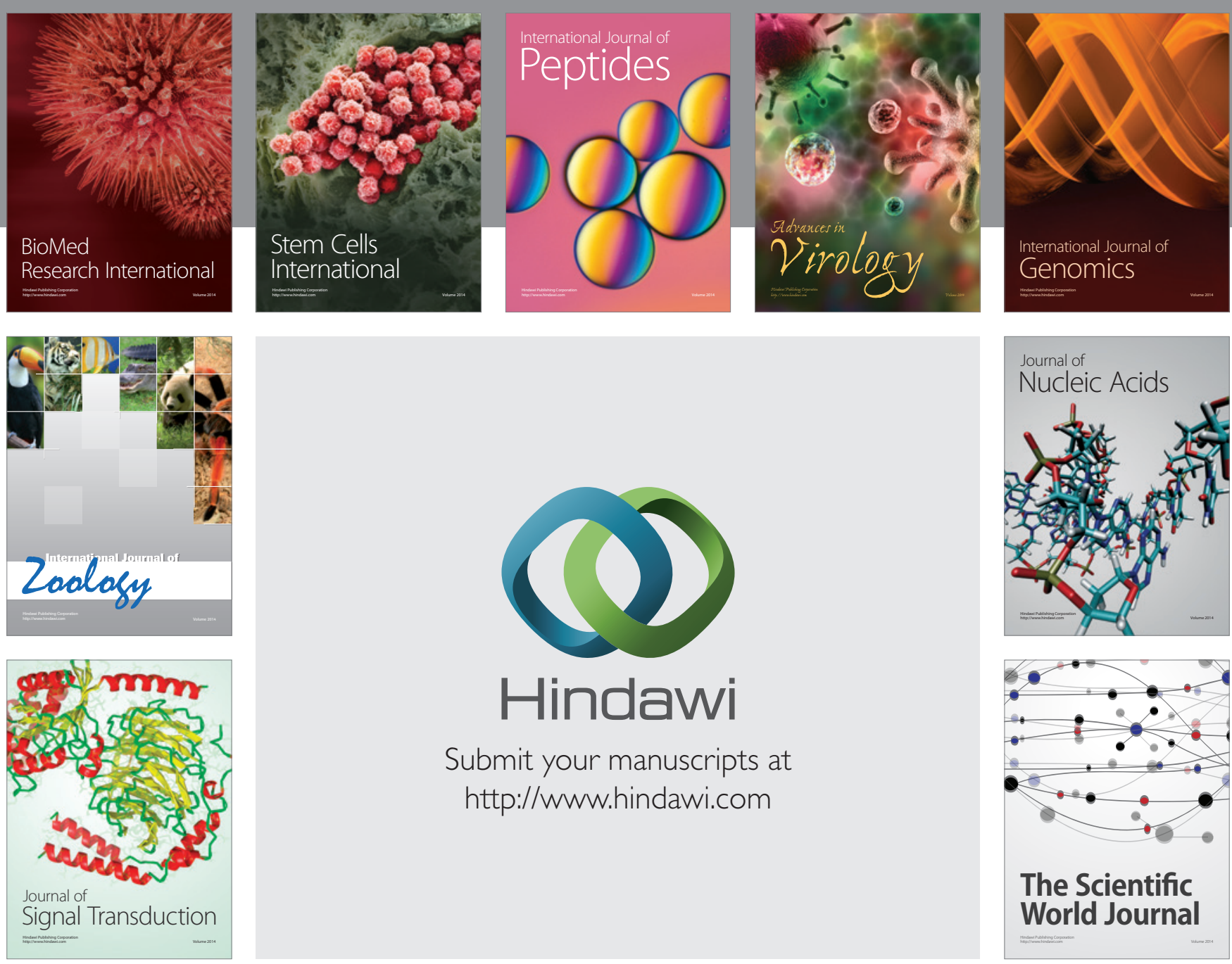

Submit your manuscripts at

http://www.hindawi.com
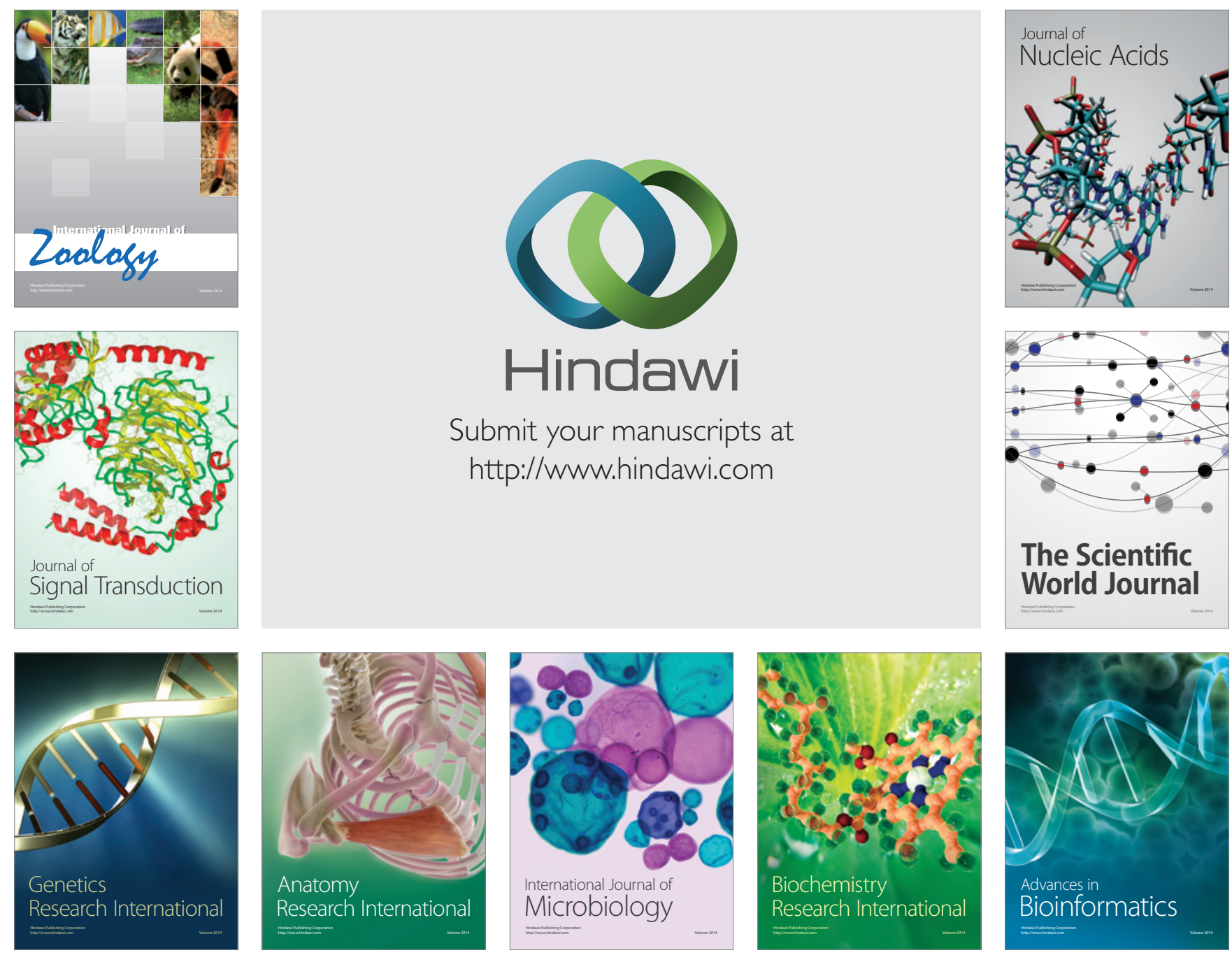

The Scientific World Journal
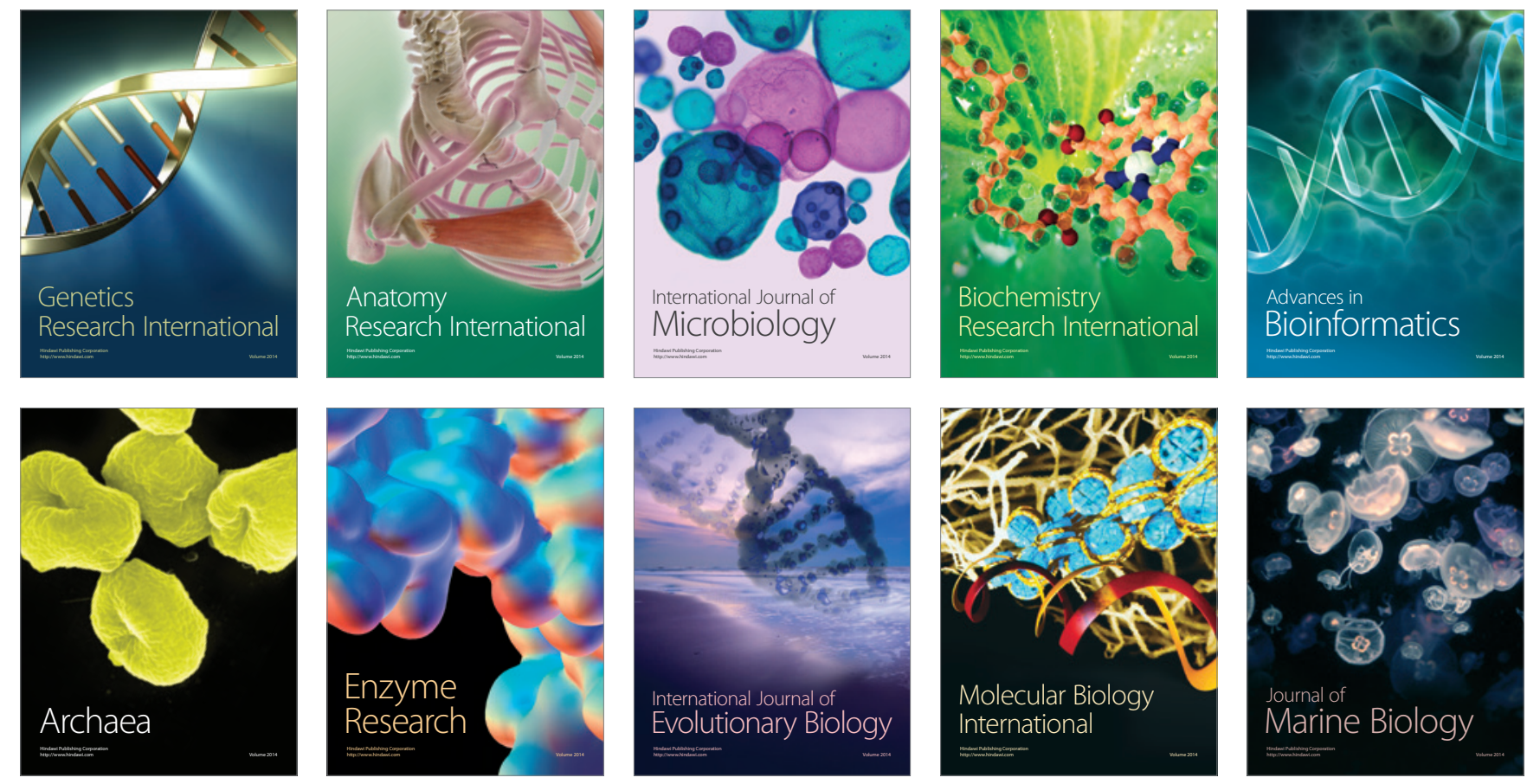